\title{
The method of the phase control of the electrical installation during geodynamic monitoring
}

\author{
$N V$ Dorofeev $^{1, *}, A V$ Grecheneva $^{1}, O R$ Kuzichkin $^{1}, R V$ Romanov $^{2}$, and $D I$ Surzhik $^{2}$ \\ ${ }^{1}$ Belgorod State University, 85 Pobedy St, Belgorod, 308015, Russia \\ ${ }^{2}$ Vladimir State University, 87 Gor'kogo St, Vladimir, 600000, Russia
}

\begin{abstract}
The paper substantiates the application of the phase control of the multipolar equipotential electrical installation to improve the geodynamic sensitivity of the geoelectric control method in geodynamic and geotechnical monitoring systems. The principle of the control of the source of sounding signals and the detection of geodynamics of the geological environment is shown in article. In this work is proposed the device on base of the phase modulator to eliminate the shortcomings of existing devices of the controlling of the phase of sound geoelectrical signals. The developed device contains two amplitude limiters, the integrating unit, the adder, the master oscillator and the amplitude modulator. In practice the application of the proposed device allow to obtain the technical and the economic efficiency by simplifying of the circuit of the generation of the signal with the phase-modulation, by increasing the linearity of the modulation characteristic, and by generating of the signal with the phase-modulate on output of the device.
\end{abstract}

\section{Introduction}

In the zone of the location of technical and life support facilities of natural-technical systems (NTS) geodynamic variations of the geological environment (in particular, variation its deformed state) are associated with regional geodynamics and the current movement of rocks, which are determined by technogenesis and climatic factors. It should be noted that the geological environment is subject to variations not only in seismically active and mountainous areas, but also on stable tectonic platforms. Differences are only in speeds and levels of variations [1,2]. Geodynamic variations of the environment have ubiquitous and everyday character. They are manifested with varying intensity and they depend on the conditions of technogenesis in the NTS, which is determined by the structure and the intensity of the natural-technogenic load on the environment. Natural-technogenic processes pass with different speeds and intensity. They influence on the geological environment. Underestimation of their danger can lead to a loss of stability of the NTS and further catastrophic consequences. Currently, due to the intensive development of territories, consideration of modern geodynamic processes, which occur in the NTS, are relevant. The estimate and the forecast of catastrophic variations of the geological environment are relevant too. Note that catastrophic variations can be triggered not only by sudden catastrophic variations, but also by "slow" processes, such as landslide and karst-suffusion processes [3].

\footnotetext{
${ }^{*}$ Corresponding author: dorofeevnv@yandex.ru
} 
At present, systems of the geotechnical and the geodynamic monitoring are used to solve problems of the protecting of life-supporting and technogenic objects from possible catastrophic scenarios, as well as for control of the bearing capacity of soils during the life cycle of objects and the NTS on the whole [4-6]. They allow to lead the estimate of geomechanical variations in the geological environment, with a view to the formation of predictive estimate of the possibility of the emergence of technogenic catastrophes. The use of multipolar electrical installations of equipotential type has shown special efficiency in solving this problem. In these installations, the amplitude-phase parameters of the control signals of the electrical installation perform role of informative parameters. The control of the installations perform by the measured signals of the geodynamic shift of equipotential lines of the created geoelectric field [7].

The purpose of this work is to justify the application of the phase control of the multipolar equipotential electrical installation to improve the geodynamic sensitivity of the geoelectrical method of the control.

\section{The phase positioning of the multipolar electrical installation during geodynamic control}

For geodynamic control the multipolar equipotential electrical installation was used, which was developed jointly with the Institute of Physics of Earth of the Russian Academy of Sciences [8]. It is designed to track the geodynamics of near-surface heterogeneities in cases where it is necessary to provide increased sensitivity to special variations of the object of research. High efficiency is achieved by increasing the sensitivity of the measuring system, the initial installation and the operational positioning of the installation by controlling sources of sounding signals. The principle of the work is based on the fact that in the researching medium a spatially distributed signal is generated by the source of sounding signals in accordance with the principle of superposition. The sounding signal generates summary zero signals in the measuring sensors of the geoelectric field. The geodynamic of the researching object is determined by the displacement of fictitious sources, which leads to an imbalance in the measuring system and the registration of the corresponding vector of the signal in it:

$$
\Delta X_{i}=\sum_{j=1} \sum_{k=1}\left(X_{j k}(t)-X_{j k}^{0}(t)\right), \Delta Y_{i}=\sum_{j=1} \sum_{k=1}\left(Y_{j k}(t)-Y_{j k}^{0}(t)\right)
$$

The displacement of fictitious sources can be represented by the model of the additivemultiplicative class [9]:

$$
\begin{aligned}
& X_{j k}(t)=\Delta_{j k}^{x}(t, \bar{\alpha})\left(1+\xi_{j k}^{x}(t)\right)+X_{J K}^{0}(t), \\
& Y_{j k}(t)=\Delta_{j k}^{x}(t, \bar{\alpha})\left(1+\xi_{j k}^{y}(t)\right)+Y_{j k}^{0}(t)
\end{aligned}
$$

where $\Delta_{j k}^{x}(t, \bar{\alpha}), \Delta_{j k}^{y}(t, \bar{\alpha})$ - the signal of displacement of fictitious sources taking into account the dependence on the vector of geodynamic parameters of the object of the control; $\bar{\alpha}, X_{j k}^{0}(t), Y_{j k}^{0}(t)$ - the trend of the bias signal; $\xi_{j k}^{x}(t), \xi_{j k}^{y}(t)$ - random stationary processes which characterize the effect of noise-producing factors.

In this case, the signals of the control of the initial installation and the positioning of measuring geoelectrical systems are formed by the phase method in accordance with:

$$
\bar{\varphi}_{i}\left(t_{0}\right)=F_{U}\left(\boldsymbol{M}_{S i}, \bar{\varphi}^{*}\left(t_{0}\right)\right)
$$

where $F_{U}$ is the forming functional of the initial positioning by the phase vector of the control $\bar{\varphi}^{*}(t)$ of the system of the spatio-temporal processing of the data of the control at the time of launch $t=t_{0}, \boldsymbol{M}_{S i}$ is the vector of the parameters of the model. 
In the future, the measuring geoelectric system operates directly in semi-automatic mode by the following algorithm:

$$
\bar{\varphi}_{i}(t)=\bar{\varphi}_{i}\left(t_{0}\right)+\Delta \varphi\left(\boldsymbol{M}_{S i}, \bar{\alpha}\right)+F_{U}\left(\Delta \boldsymbol{M}_{S i}, \bar{\varphi}^{*}(t)\right),
$$

where $\Delta \varphi\left(\boldsymbol{M}_{S i}, \bar{\alpha}\right)$ - the current control of the positioning of the electrical installation by the vector of geodynamic variations $\bar{\alpha} ; \Delta \boldsymbol{M}_{S i}$ - the correction of the model.

\section{The principle of functioning of the phase modulator}

The proposed phase modulator allows the installation of phase signals of the control with high accuracy in systems of the geodynamic control. The disadvantages of the previously used devices of the control of the phase of the sounding geoelectrical signals are the complexity of the devices as a whole, the complexity of providing linearity during commutating charging capacitors, the complexity of ensuring the stability of levels of the compare, the need for additional filtering to extract (form) the signal with phase modulation [10].

The block diagram of the proposed device shows on the figure 1. The device contains the amplitude modulator - 1 , the adder - 2, the master oscillator - 3 , the first amplitude limiter - 4, the integrating device -5 and the second amplitude limiter -6 . The input of the device is the first input of the amplitude modulator 1, and the output is the output of the second amplitude limiter 6 . The harmonic signal of the master oscillator 3 is converted by the first amplitude limiter 4 in a rectangular waveform that is fed to the second input of the amplitude modulator 1, where its multiple are with the input signal, that is applied to the first input of the amplitude modulator. In the general case the input signal contains a variable and a constant component (offset)

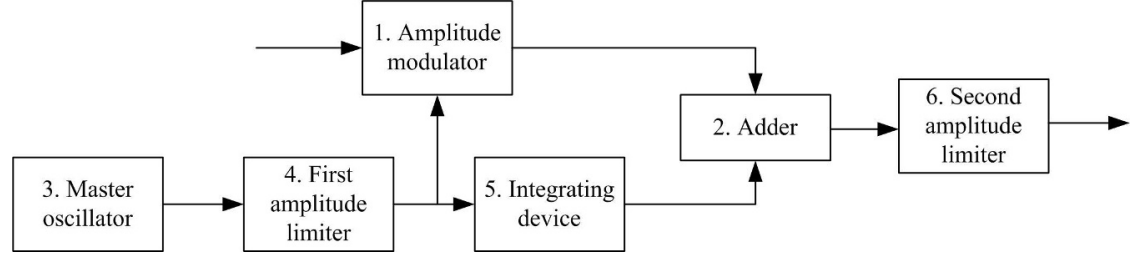

Fig. 1. Flowchart for controlling the phase of sounding signals.

The amplitude-modulated signal thus obtained has a rectangular carrier and is fed to the first input of the adder 2 from the amplitude modulator 1 . At the second input of this adder, the signal of tha triangular waveform is fed shifted by a quarter of the period relative to the signal at the first input. The integrating device 5 is used to form and shift the signal of the triangular waveform. At the output of the adder 2, there is a signal of the sum of the amplitude modulated signal with the rectangular carrier and the signal of the triangular waveform. The signal of this sum lags in time during the pass of the zero level relatively to the signal of the rectangular waveform from the output of the limiter 4 . From the output of the adder 2, the signal is applied to the input of the second amplitude limiter 6 , at the output of which the phase modulated signal of the rectangular shape is formed (with spectral components modulated in phase).

Essential distinctive features of the consideration device are:

- the use of the first amplitude limiter in it. This is achieved the simplicity of the circuit realization of the amplitude modulator (a conventional electronic key) and the obtaining of the amplitude modulated signal with the carrier of the rectangular waveform;

- the use of the integrating device of high (2nd and higher) order [11,12] with the purpose of forming the triangular waveform with increased linearity of growth. Namely, the 
rectangular carrier and the linearly variation signal of the triangular waveform provide the linear variation of time shifts during summing signals;

- the use of the second amplitude limiter makes it possible to obtain direct signal formation with phase modulation at the output of the device;

- the device does not contain commutated energy-intensive elements (capacitors) in comparison with the devices used. It increases the linearity interval of the conversion over the period and simplifies the provision of this linearity.

Figure 2 shows the waveforms of the signals at the inputs and outputs of the phase modulator: (a) - the signal at the input of the device, witch contain the constant component (displacement) $U_{0}$ and the linearly varying signal with the period $T_{c}$ and amplitude $U_{c} \leq U_{o}$; (b) - the modulating signal of the square-wave at the output of the first amplitude limiter 4 and the second input of the modulator 1 with the period $T_{\mathrm{P}} ;$ (c) - the amplitude modulated signal $U_{A M}$ with the rectangular carrier at the output of the amplitude modulator 1; (d) - the signal of triangular shape with amplitude $U_{P} \geq U_{0}+U_{c}$ at the output of the integrating device 5; (e) - the signal at the output of the adder 2, which is equal to the sum of the signals in figures 2(c) and 2(d), $U_{\Sigma}=U_{A M}+U_{P}$ it acquires a time lag $\Delta t$, depending on $U_{c}$ the signal of figure 2(c) along the zero-level axis; (f) - the formed signal of the rectangular waveform with the phase modulation at the output of the second amplitude limiter 6 (dashed for clarity, a constant-shift signal is shown for the case $U_{c}=0$, its amplitude is conventionally increased).

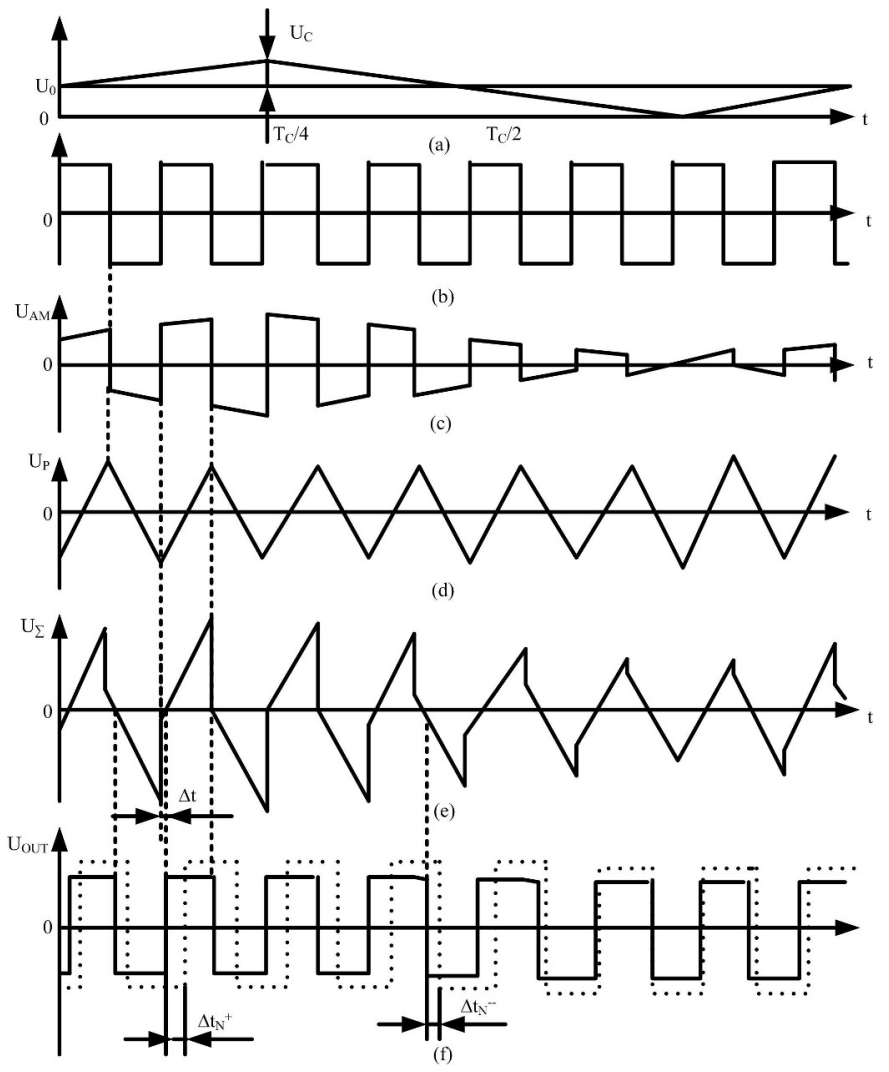

Fig. 2. Diagrams of the operation of the phase modulator. 
As follows from figure 2 , in the first harmonic of the output signal the maximum phase shift reaches values $\pm \pi / 2, \pm \pi / 4$ depending on the presence of the bias $U_{0}$. The time shifts $\Delta t_{N}^{+}$and $\Delta t_{N}^{-}$are directly proportional to the magnitude of the signal, as follows from the diagrams in figure 2. More precisely, the time shifts are determined by the equations:

$$
\frac{t_{O}-t_{N}^{+}}{T_{P} / 4}=\frac{U_{C}}{U_{P}}\left[1+\frac{T_{P}}{T_{C}}\left(4 N-3+\frac{U_{O}}{U_{P}}\right)\right] /\left(1-\frac{U_{C} T_{P}}{U_{P} T_{C}}\right), t_{O}=\frac{T_{P} U_{O}}{4 U_{P}} .
$$

The foregoing relations are valid for positive half-waves of the amplitude-modulated signal, which are in phase with a decaying half-wave $d U_{P} / d t<0$ of the signal of the triangular waveform, and $d U_{C} / d t>0$. For the case $d U_{C} / d t<0$ in the above equations (5), the signs must be reversed before $U_{C} / U_{P}$ the relations. The same change of signs must be made when the phase of the amplitude-modulated signal changes to the reverse one.

\section{Conclusion}

The use of the proposed device allows to simplify circuits of the generation of the signal with the phase-modulation, to increase the linearity of the modulation characteristic, and to directly generate a signal with phase modulation at the output of the device. These advantages determine the technical and economic effect of the proposed modulator. When $T_{P}<<T_{c}$ the linear dependence of time shifts on the value of the signal ensures linearity of the phase shifts of the spectral components of the signal and the direct formation of the signal with phase modulation at the output of the device.

\section{Acknowledgments}

The work was carried out as part of the research on the project, support by the Ministry of Education and Science of the Russian Federation No. 5.3606.2017/PCH.

\section{References}

1. Yi N P, Zhang X G and Ma F R 2009 Int. Conf. on Management and Service Science 22-23 Sept. (Wuhan: IEEE) pp 1-4

2. Grecheneva A, Eremenko V, Kuzichkin O and Dorofeev N. 2017 CEUR Workshop Proc. 28 Aug.- 5 Sept. 2016. Vrnjachka Banja vol 1839 (Vrnjachka Banja) pp 74-83

3. Tatevian S K, Attia G F, Abou-Aly N, Ghoneim R and Hegazy M 2014 NRIAG $J$. of Astron. and Geophys. 3 46-52

4. Zanutta A, Negusini M, Vittuari L, Cianfarra P, Salvini F, Mancini F, Sterzai P, Dubbini M, Galeandro A and Capra A 2017 J. of Geodynamics 110 31-42

5. Lebedev M 2016 Proc. Engin. 12-15 Sept. 2016 St. Peterburg vol 165, ed. S Alpatov, O Prentkovsky, R L Sterling, D Kaliampakos (St Peterburg: Elsevier) pp 448-54

6. Bykov A, Kuzichkin O, Dorofeev N and Koskin A 2017 Proc. Comp. Sci. vol 103, ed. Diveev A, Zelinka I, Kusiak A, Nikulchev E (Moskow: Elselvier) pp 253-9

7. Kuzichkin O, Grecheneva A, Mikhaleva E, Dorofeev N and Maxim B 2017 J. of Engin. and Appl. Sci. 12 6858-63 
8. Dorofeev $\mathrm{N}$ and Kuzichkin O 2016 2nd Int. Conf. on Industrial Engin., Application and Manufacturing (Chelyabinsk: IEEE) pp 1-4

9. Tsaplev A, Dorofeev N and Kuzichkin O 2015 IEEE 8th Int. Conf. on IDAACS 24-26 Sept. Vol 1 (Warhaw: IEEE) pp 38-41

10. Lu R, Zhou B and Gao W 2009 9th Int. Conf. on Electronic Measurement and Instruments 16- 19 Aug (Beijing: IEEE) pp 2-486-90

11. Prabaharan N, Rini Ann Jerin A, Palanisamy K and Umashankar S 2017 Energy Proc. vol 138, ed. Waewsak J, Sangkharak K, O-Thong S and Gagnon Y (Elselvier) pp 117783

12. Hung-Chun C 2014 Microelectonics J. 45 634-47 